\title{
Effect Of Relay Nodes On End-to-end Delay In Multi-hop Wireless Ad-hoc Networks
}

\author{
Raja Vara Prasad Yerra \\ Electrical Engineering Department \\ Indian Institute of Technology Hyderabad \\ Hyderabad, India \\ Email: ee10p004@iith.ac.in
}

\author{
Rajalakshmi P \\ Electrical Engineering Department \\ Indian Institute of Technology Hyderabad \\ Hyderabad, India \\ Email: raji@iith.ac.in
}

\begin{abstract}
Channel access delay in a wireless adhoc network is the major source of delay while considering the total endto-end delay. Channel access delays experienced by different relay nodes are different in multi-hop adhoc network scenario. These delays in multi-hop network are analysed in the literature assuming channel access delays are independent and are of same magnitude at all the nodes in the network. In this work, the endto-end delay in a multi-hop adhoc network is analysed taking into account the silent relay nodes. Along with silent relay node effect, Channel access probability (p), transmission radius ( $r$ ) analogous to transmit power, network throughput and density of nodes are the other factors considered for the end-to-end delay analysis. Effect of network parameters along with silent relay nodes on end-to-end delay is found to be considerably high compared to the previous literature results. Given a bound on end-to-end delay with percentage of silent relay nodes, throughput, node density requirements for a multi-hop adhoc network, optimal ranges of transmission radius and channel access probability can be obtained from the proposed analysis. End-to-end delay increases with silent relay nodes along with transmission radius(r), channel access probability(p), node density and throughput. It is clear from the analysis, that the effect of silent relay nodes on endto-end delay cannot be ignored to maintain certain Quality of service (QoS) metrics for the multi-hop wireless adhoc network.

Index Terms-silent relay nodes, channel access probability, node density, end-to-end delay.
\end{abstract}

\section{INTRODUCTION}

Adhoc networks can communicate without fixed infrastructure. Ease and less time for deployment are the main advantages of adhoc networks. The adhoc networks are used in very diverse region of application from biological spheres to ubiquitous computing. Wireless sensor based adhoc networks have low bandwidth and more channel access delays. Delay is considered to be one of the main quality of service (QoS) metric for time constrained applications in adhoc networks. Wireless Sensor networks is one of major applications nowadays as they operate mostly in adhoc mode. Diverse delay requirements are required to support heterogeneous sensor network applications. Applications like detection of fire in forest, seismic activity need delay differentiation for best use of the measurements obtained by sensors.

\section{A. Delay in wireless network:}

Delays in a wireless network can be due to three main sources

1) Delay due to multi-hop : Message traverses several hops before reaching destination in multi-hop networks. Transmission power is analogous to transmission radius (r) so Low power operation of nodes in battery operated sensor networks also increases the number of hops in the network which is another reason for larger delays. Transmission power can be increased to reduce the hops but not at the cost of more interference with the neighbouring nodes.

2) Channel access delay: The channel access mechanisms are mostly CSMA/CA based contention access in wireless networks. Collisions creates additional exponential delays in the network. Channel access delays depends on throughput of each node, node density and number of nodes in the network and transmission power.

3) Aggregation and queuing delays at intermediate nodes : Aggregation and compression in adhoc networks is used to reduce the redundancy of the messages thereby reducing the channel access delays. Aggregation and compression function of throughput and protocols at intermediate nodes can lead to large delays due to processing delays before transmission.

These three sources of delay are tightly coupled and should be optimized considering all the above factors. The delay experienced by a message due to network throughput, node density, silent relay nodes and transmission power is analysed using simple models to understand trade-off among them in this paper. As a result, understanding the relationship between delay and the network parameters is an important first step in providing delay differentiation. Aggregation and queuing delays are ignored in this paper.

Optimal network throughput is studied considering the effect of transmission power in [4] and is shown that it is obtained at the lowest transmission power that allows connectivity. The interference caused is very little with smaller transmission ranges. Lower transmission power increases number of hops (n) to the destination which intern increases the delay and scales down the throughput to $1 / \sqrt{(} n)$ according to [3]. Trade-off exists between delay experienced and achievable throughput in the network as shown in [2] and in [8], but the 


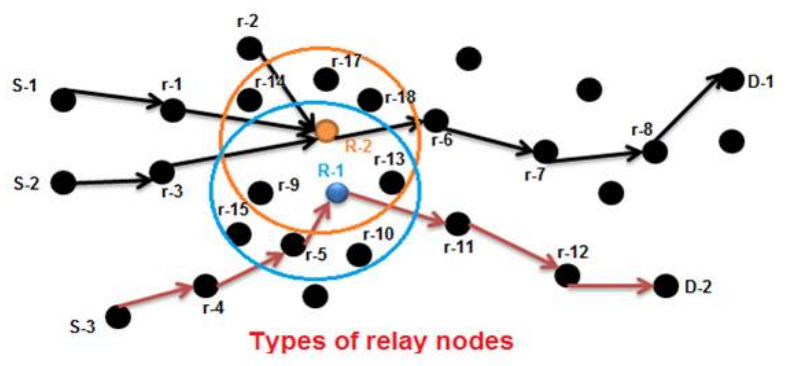

Fig. 1. Pictorial representation of types of relay nodes in multi-hop adhoc network

effect of collisions and channel access mechanism are ignored in both the analysis.

MAC layer protocols $[9,11,12]$ and power control protocols $[1,5-6,8]$ have been proposed in the literature to provide better throughput and energy efficiency. The CSMA/CA protocol is the most common MAC layer protocol in the 802.11 standard of the IETF. The basic access mechanism, called the Distributed Coordination Function (DCF), is a Carrier Sense Multiple Access with Collision Avoidance mechanism (CSMA/CA). DCF initializes a random countdown timer and senses the medium. Contention in the medium causes more end-to-end delay due to exponential back-off. In multi-hop adhoc networks nodes forward data to sink using relay nodes if it is not in direct transmission range. End-to-end delay in a multihop network is mainly a function of channel acces delay at each realy node. Channel access probabilities in a multi-hop network is assumed to be independent and same in magnitude for all relay nodes in the literature which is not valid for all types of relaying nodes. Relay nodes of different types and the possible reasons for delay is mentioned in next subsection. A silent relay node concept is introduced in this paper which is defined and illustrated with figures in the next subsection.

\section{B. Silent relay node}

Networks with high node density have to follow similar to intereference model in [4] to reduce the interference. The interference model is similar to Protocol model in [4] when one node is transmitting the nodes that should remain silent is given as shown in the equation-1 and equation-2. Silent relay node is the relay node that will remain silent during other nodes transmission when it is present in other nodes transmission region.Three types of relay nodes are considered for analysis as shown in the Figure-1. The node R-2 shown in the Figure-1 is common relay node for three multi-hop routes. $\mathrm{R}-1$ relay node is single path relay node. Both R-1 and R-2 are silent relay nodes and remaining relay nodes are normal relay nodes which just forward data. In this paper total end-to-end delay is analysed with silent relay nodes and silent relay node with multiple multihop paths such as R-2 are ignored for the sake of simplicity.

Nodes in a multi-hop network can transmit data with a certain transmission power to reach the next hop. The nodes in this transmission region (r) can hear this data transmission. The nodes in this transmission region should remain silent when one node is transmitting. Nodes r-9,13,14,17,18, R-1 should remain silent for R-2 relay node transmission and nodes $\mathrm{r}$ $5,9,10,13,15, \mathrm{R}-2$ should remain silent for R-1's transmission. If the nodes $i$ and $j$ are within a distance $r$ of each other and $\mathrm{k}$ is any other node

$$
\begin{gathered}
d(i, j) \leq r \\
d(i, k) \leq(1+\Delta) r
\end{gathered}
$$

for some $(\Delta>0)$

The first equation indicates that node $\mathrm{j}$ to be within the transmission range of node $i$. The second equation requires a $(1+\Delta)$ r neighborhood of node $\mathrm{i}$ to be silent for successful transmission. $\Delta=0$ is the condition for only one hop neighbor nodes to be silent but as shown in the Figure-1 the relay nodes R-1 and R-2 of different multi-hop routes are in each others transmission range. The nodes that should remain silent for R-1's transmission is r-5,r-9,r-10,r-13,r-15 and R-2. In similar way the nodes that should remain silent for R-2's transmission is r-9,13,14,17,18 and R-1. Nodes r-14,r-17,r-18 should remain silent for R-1's transmission eventhough there are not in the transmission region of R-1. This is because the relay node R-2 which is in the silent region cannot accept packets from these nodes. The end-to-end delay increases due to silent R-2 node eventhough the nodes r-14,r-17,r-18 are not in the transmission region of R-1 virtually making second hop neighborhood to be silent $(\Delta=1)$. The effect of silent relay nodes $(\alpha)$ along with network parameters like node density $(\Lambda)$, throughput $(\lambda)$, and channel access probability(p) is discussed in this paper. Percentage of silent relay nodes is assumed to be $\alpha$ among the total relay nodes to find the end-to-end delay.

The rest of the paper is organized as follows. Section-II describes the system model and the effect of silent relay nodes on end-to-end delay is discussed. Section-III presents the simulation results and Section-IV concludes the paper.

\section{SySTEM MOdeL}

The end to end delay in multi-hop adhoc network is mainly due to channel access delay and queuing delays. In this paper effect of silent relay node delay is considered on the end-toend delay of multi-hop network. Effect of network parameters on the end-to-end delay is analysed in detail considering tradeoff between parameters. The parameters considered for analysis are transmission radius ( $r$ ), channel access probability (p), node density $(\Lambda)$ and percentage of silent relay nodes $(\alpha)$. Expected Channel access delay is derived in [13] given as in equation-3. Where $f$ is the neighbourhood radius as given in equation- 4 . The total end to end delay for a message to travel L units away is also derived in [13] as given in equation-6.

$$
\begin{gathered}
E\left[d_{c}\right]=\frac{e^{-\Lambda \pi f^{2}}}{p}\left[e^{\frac{\Lambda \pi f^{2}}{1-\frac{p \lambda D}{r}}}-1\right] \\
f=(1+\Delta) r
\end{gathered}
$$




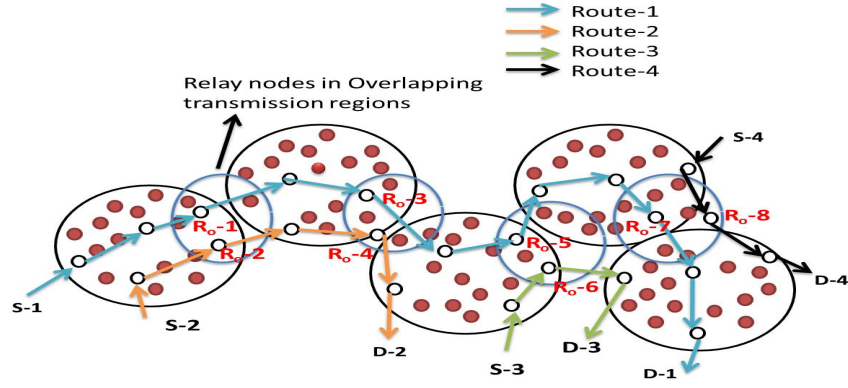

Fig. 2. Topology of nodes with relay nodes in overlapping transmission region

$$
\begin{gathered}
d_{t}=\sum_{j=1}^{n} d_{c}(j) \\
E\left[d_{t}\right]=\frac{L e^{-\Lambda \pi f^{2}}}{p * r}\left[e^{\frac{\Lambda \pi f^{2}}{1-\frac{p \lambda D}{r}}}-1\right]
\end{gathered}
$$

$\Delta$ is number of neighbour hops to be silent during transmission. Nodes to be silent is taken as 2 hops for overlapping regions.

$\Lambda$ is the node density

\section{A. Effect of silent relay nodes delay on end-to-end delay}

The channel access delay in the single hop neighbourhood is considered in [13]. The underlying assumption in [13] is that channel access delay at each hop is independent and same magnitude. Relay nodes in overlapping transmission region remains silent for other relay nodes transmission. The percentage of relay nodes that are silent in a multi-hop route decides the amount of end-to-end delay. The percentage of number of relay nodes in overlapping transmission regions in multi-hop route to the destination is considered in calculating the end-to-end delay analysis. The effect of silent relay nodes is explained in more detail in next paragraph with help of pictorial representation of nodes as shown in the Figure-2.

Route- 1 is between nodes S-1 and D-1; Route- 2 is between nodes S-2 and D-2; Route-3 is between nodes S-3 and D-3; and Route-4 is between nodes S-4 and D-4

An example node distribution of adhoc wireless network is shown in the Figure-2. Four simultaneous multi-hop routes with different number of relay nodes per route is shown in the Figure-2. Nodes in white colour shown in the Figure-2 are relay nodes of multi-hop routes where each route indicated with different colour arrows. Two types of relay nodes are shown in the Figure-2. Some of the relay nodes are present in bigger circles and some of them in the smaller circles. Relay nodes in the bigger circles are not in the vicinity of any other relay nodes where as the relay nodes shown in the smaller circle are in the one-hop transmission range of other relay node of different route. For example the $R_{O}-1$ is in the transmission range of $R_{o}-2$ of route- 2 . The assumption here is that preceding relay node of $R_{O}-1$ cannot hear from $R_{O}-2$ and vice-versa.

The delay for normal relay nodes is same as mentioned in literature [13] derived in the equation-6. For example if we consider relay nodes $R_{o}-1$ and $R_{o}-2$ both are in one others transmission region, relay node $R_{O}-1$ should remain silent for $R_{o}-2$ transmission and vice versa. Average end-to-end delay increases for each route as the relay nodes are in the 2 hop silent region $(\Delta=1)$ to minimize the interference. The end-toend delay considering silent relay nodes is derived in equation13. The percentage of silent relay nodes $(\alpha)$ is formulated as shown below.

from the Figure-2 in multi-hop route-1 (S-1 to D-1)

Total relay nodes $(\mathrm{T})=10$

Number of relay nodes with $\Delta=1$ ( 2 hop neighbour hood silent nodes) for route- $1(\mathrm{t})=4$

Hence the percentage $(\%)$ of silent relay nodes for any route is as below

$$
\alpha=(t / T)
$$

Thus $\alpha$ for four multi-hop routes route-1,2,3,4 shown in Figure-2 are 0.4, 0.4, 1, 1 respectively. $\alpha$ is used in calculating total end-to-end delay. End-to-end delay of relay nodes in the bigger circles are considered in equation- 8 with $\Delta=0$. Endto-end delay of silent relay nodes with $\Delta=1$ is given in equation-10. Total end-to-end delay of all relay nodes is given in equation- 13 by combining equations- 8 and equation- 10 .

$$
\begin{gathered}
E_{1}\left[d_{t}\right]=\frac{(1-\alpha) e^{-\Lambda \pi f_{1}^{2}}}{p * r}\left[e^{\frac{\Lambda \pi f_{1}^{2}}{1-\frac{p \lambda D}{r}}}-1\right] \\
f_{1}=(1+\Delta) r
\end{gathered}
$$

$\Delta=0$ for relay nodes in non overlapping transmission regions

$$
\begin{gathered}
E_{2}\left[d_{t}\right]=\frac{\alpha e^{-\Lambda \pi f_{2}^{2}}}{p * r}\left[e^{\frac{\Lambda \pi f_{2}^{2}}{1-\frac{p \lambda D}{r}}}-1\right] \\
f_{2}=(1+\Delta) r
\end{gathered}
$$

$\Delta=1$ for relay nodes in overlapping transmission regions

Total end-to-end delay is obtained by combining the equation- 8 and equation- 10

$$
E\left[d_{t}\right]=E_{1}\left[d_{t}\right]+E_{2}\left[d_{t}\right]
$$

$E\left[d_{t}\right]=\frac{(1-\alpha) e^{-\Lambda \pi f_{1}^{2}}}{p * r}\left[e^{\frac{\Lambda \pi f_{1}^{2}}{1-\frac{p \lambda D}{r}}}-1\right]+\frac{\alpha e^{-\Lambda \pi f_{2}^{2}}}{p * r}\left[e^{\frac{\Lambda \pi f_{2}^{2}}{1-\frac{p \lambda D}{r}}}-1\right]$

Thus end-to-end delay experienced by a message in a wireless adhoc networks is derived taking into effect the percentage of silent relay nodes $(\alpha)$ 


\section{SIMULATION RESULTS}

The total end-to-end delay in a multi-hop network is primarily dependent on channel access delay at each relay node. Channel access delay derived in [13] is a function of channel access probability(p), transmission radius(r)/power and other network parameters like node $\operatorname{density}(\Lambda)$ and throughput $(\lambda)$. The channel access delay with variation in distance ( $r$ ) for different channel access probability is simulated as shown in Figure-3. The optimal minimum distance for each channel access probability is different. The channel access delay $\left(E\left[d_{c}\right]\right)$ for each given channel access probabilities for equation-3 is as shown in the Figure-3. This region indicates that channel access delay can be kept low with a given $\mathrm{p}$ value upto certain transmission radius where the delay again start increasing. This transmission radius range is different for different $p$ values as shown in the Figure-3. For example if we take bound of 15 units on channel access delay the distance( $r$ ) is 0.6 for $p=0.4$ and 0.7 for $p=0.15$. Increase in channel access probability(p) will increases the contention among nodes. To meet the same delay requirements transmission power/ radius should be reduced to reduce the number of contending nodes. End-to-end delay in a multi-hop network is given in the

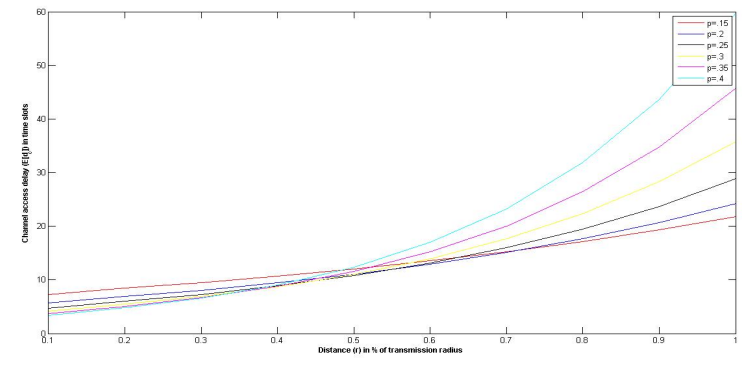

Fig. 3. Channel access delay with variation in distance(r), $\Lambda=100, \lambda=$ $0.025, \Delta=0$ for set of channel access probabilities(p)

equation-6, where the channel access probability is assumed independent at each hop so the end-to-end delay is L/r hop times of channel access delay. This assumption is not valid as explained in the previous sections and a new end-to-end delay equation is derived as shown in the equation-13. End-to-end delay is simulated for both cases with and without silent relay nodes as shown in the Figure-4. The end-to-end delay is high initially and has minimum delay region at the middle and starts increasing at the end as shown in the Figure-4(a). The total end-to-end delay is effected considerably after considering the silent relay nodes compared to the previous literature results as shown in the Figure-4(a). The slope of curves are steeper in in Figure-4(b) with silent relay nodes. The transmission distance (r) for which the end-to-end delay is minimum also reduced drastically. The distance range for channel access probability of $p=.15$ is $[00.55]$ and $[0,1]$ for with and without silent relay nodes respectively. Considering twenty percentage of silent relay nodes among total relay nodes the end-to-end delay increased drastically as the
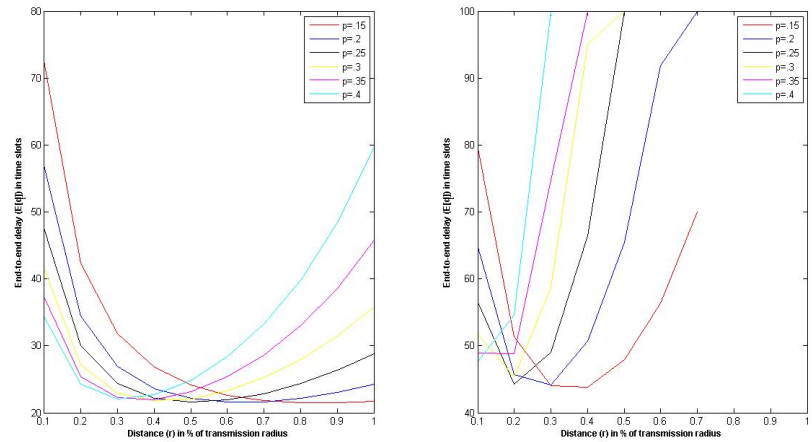

Fig. 4. End-to-end delay with variation in distance(r) a) $\Lambda=100, \lambda=$ $0.025, \Delta=0$ b) $\Lambda=100, \lambda=0.025, \alpha=0.2$ and $\Delta=1$ for set of channel access probabilities $(\mathrm{p})$

number of nodes to remain silent increased to 2 hops. The transmission distance(r) for a given end-to-end delay of 50 units and $\mathrm{p}=0.15$, reduced to 45 percentage of the previous case where there are no silent relay nodes. The distance (r) for higher probabilities is still decreasing as the silent relay nodes are adding further delay to the contending nodes in the region.
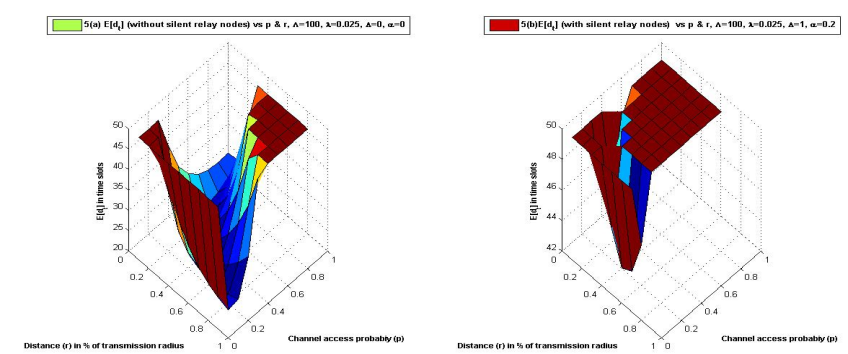

Fig. 5. 3D plot of end to end delay with variation in distance(r)and channel access probability(p) a) $\Lambda=100, \lambda=0.025, \Delta=0$ without silent relay nodes b) $\Lambda=100, \lambda=0.025, \Delta=1, \alpha=0.2$ with silent relay nodes

End-to-end delay is a function of other network parameters as shown in the equation-13. Total end-to-end delay is analysed with respect to changes in network parameters distance $(\mathrm{r})$, channel access probability (p) and percentage of silent relay nodes $(\alpha)$ while $\Lambda$ and $\lambda$ are fixed to 100 and 0.025 as shown in the Figure-5. End-to-end delay variation with respect to simultaneous network parameters $\mathrm{p}$ and $\mathrm{r}$ is shown as $3 \mathrm{D}$ plot. The $3 \mathrm{D}$ surface plot looks like a valley where there is a minimal end-to-end delay region. Given a bound on end-to-end delay and fixing the network parameters optimal transmission distance(r) and channel access probability(p) ranges can be obtained. For example if the $\left[\mathrm{E}\left[d_{t}\right], \Lambda, \lambda, \alpha\right]$ are fixed to $[30,100,0.025,0.2]$, the distance (r) and channel access probabilities (p) ranges are [0.1 0.7$],\left[\begin{array}{ll}0 & 0.9\end{array}\right]$ respectively with silent relay nodes shown in Figure-5(b)and [0 0.9$]$, [0 0.9] without silent relay nodes as shown in Figure-5(b). The transmission distance(r) range reduced considerably due to 20 percent overlap region of relay nodes compared to absence of 
silent relay nodes. The neighborhood hop nodes remains silent which increases the end-to-end delay and decreases the valley region as shown in the Figure-5(b). Thus we have analysed the end-to-end delay with variation in distance (r) and channel access probability (p) in this section. In the next subsection analysis with respect to varying node $\operatorname{density}(\Lambda)$ is considered.
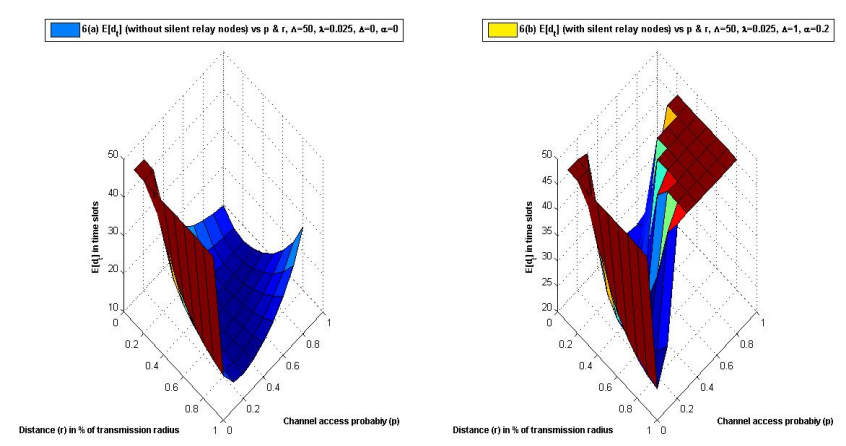

Fig. 6. 3D plot of end to end delay with variation in distance(r)and channel access probability(p)along with a) $\Lambda=50, \lambda=0.025, \Delta=0$ without silent relay nodes b) $\Lambda=50, \lambda=0.025, \Delta=1, \alpha=0.2$ with silent relay nodes
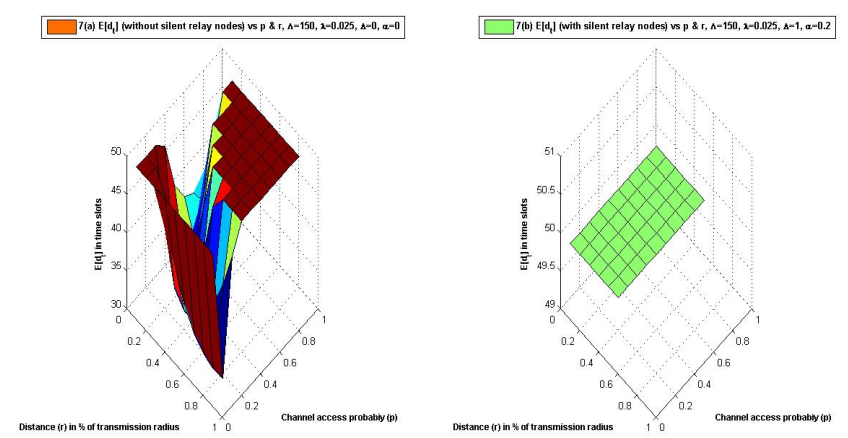

Fig. 7. 3D plot of end to end delay with variation in distance(r)and channel access probability(p)along with a) $\Lambda=150, \lambda=0.025, \Delta=0$ without silent relay nodes b) $\Lambda=150, \lambda=0.025, \Delta=1, \alpha=0.2$ with silent relay nodes

\section{A. Effect of node density $(\Lambda)$ on end-to-end delay with silent relay nodes}

Simulation results explained in previous paragraph is the analysis of end-to-end delay with variation in distance(r) and channel access probabilities (p) by fixing some of the parameters like node $\operatorname{density}(\Lambda)$, throughput $(\lambda)$ to 100 and 0.025 . The effect of varying node $\operatorname{density}(\Lambda)$ in steps of 50 and 150 with $\lambda=0.025, \alpha=0.2$ is discussed in this section. Node density is an important parameter which affects the end-to-end delay along with distance (r) and channel access probability (p). Increase in node density increases the number of nodes in the region as well as number of contending nodes. The effect of node density is with 50 and 150 to analyse the end-to-end behaviour along with $\mathrm{r}$ and $\mathrm{p}$.
Figure-6 shows the 3D plot of end-to-end delay with variation in $\mathrm{p}$ and $\mathrm{r}$ fixing $\Lambda=50$ and $\lambda=0.025$. The optimal region for minimal end-to-end delay is a valley as shown in the plot. End-to-end delay with and without silent relay nodes is plot in Figure-6(b) and Figure-6(a) respectively. From the figures the minimal delay region is very less with silent relay nodes compared to without silent relay nodes. A bound on end-to-end delay is fixed to 30 to find out optimal transmission distance. The distance $(r)$ is [0 0.9$]$ without silent

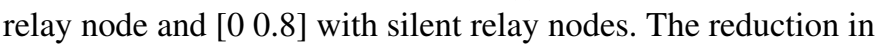
transmission distance with silent relay nodes is less compared to without silent relay nodes. This reduction in distance (r) is due to the fact that silent relay nodes are being silent most of the time than forwarding data to the next hop.

Node density $(\Lambda)$ is increased to 150 as shown in Figure-7. Simulations are carried out for both cases with and without silent relay nodes as shown in Figure-7(a) and Figure-7(b). Same end-to-end delay bound of 30 units is taken for $\Lambda=150$ also. The transmission distance (r) is [0 0.8$]$ without silent relay nodes as shown in the Figure-7(a), where as the minimal end-to-end delay starts at 50 units with silent relay nodes as shown in Figure-7(b). Thus there is no optimal transmission distance with silent relay nodes when the end-to-end delay bound is 30 units. Minimum end-to-end delay is increasing with silent relay nodes. Minimum end-to-end delay region is reducing with a steeper slope with silent relay node case as shown in the Figure-6(b) and 7(b). The observations shows that node density is a dominant factor which affects end-to-end delay. Thus effect of node density is analysed with silent relay nodes in this section. End-to-end delay with consideration of throughput effect along with silent relay nodes is discussed in the next subsection.

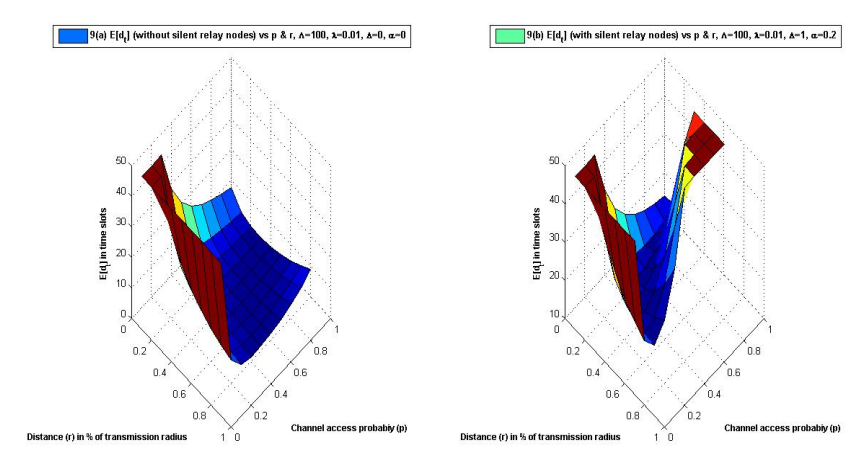

Fig. 8. 3D plot of end to end delay with variation in distance(r)and channel access probability(p)along with a) $\Lambda=100, \lambda=0.01, \Delta=0$ without silent relay nodes b) $\Lambda=100, \lambda=0.01, \Delta=1, \alpha=0.2$ with silent relay nodes

\section{B. Effect of throughput on end-to-end delay with silent relay nodes}

End-to-end delay is also a function of throughput $(\lambda)$. Analysis of throughput effect on end-to-end delay is discussed in this section. Throughput $(\lambda)$ is transmission of bits to the destination from source. These bits should be 

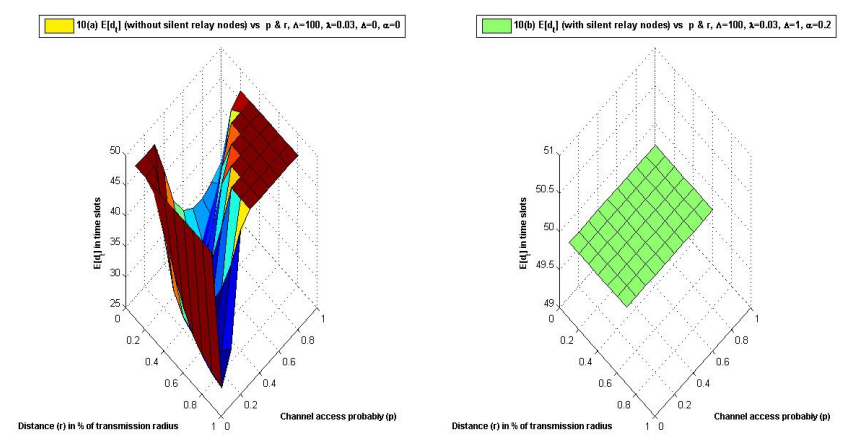

Fig. 9. 3D plot of end to end delay with variation in distance(r)and channel access probability(p)along with a) $\Lambda=100, \lambda=0.03, \Delta=0$ without silent relay nodes b) $\Lambda=100, \lambda=0.03, \Delta=1, \alpha=0.2$ with silent relay nodes

forwarded through relay nodes in a multi-hop network. If the throughput $(\lambda)$ is more the relay nodes need more time to transfer data to the destination which will create more end-to-end delay. The effect of silent relay nodes will further increase the end-to-end delay as the relay nodes supposed to forward are silent due to interference.

The total end-to-end delay with variation in distance (r) and channel access probability ( $\mathrm{p}$ ) is simulated by keeping node density $(\Lambda)$ constant at 100 and throughput is varied between .01 and .03 as shown in the Figure- 8 and Figure-9 respectively. End-to-end delay simulations show that the minimal delay region of end-to-end delay is reducing with increase in the throughput without consideration of silent relay node delay effect as shown in Figure-8(a) and Figure9(a). A bound of 30 units end-to-end delay will result in transmission distance of [ $\left.\begin{array}{ll}0 & 0.8\end{array}\right]$ and $\left[\begin{array}{ll}0 & 0.6\end{array}\right]$ respectively for with and without silent relay nodes as shown in Figure-8(a) and 8(b). Transmission distance for the same delay bound of 30 reduced to $25 \%$ of without relay nodes. In a similar fashion, when the throughput is increased to 0.03, keeping other parameters same, it is observed that the minimal end-to-end delay is 50 units, and for bound of 30 units there is no optimal distance as shown in the Figure-9(b). Increase in throughput $(\lambda)$ is reducing the minimal end-to-end delay region drastically. The optimal end-to-end delay region for $\lambda=0.03$ is considerable as shown in Figure-9(a), where as it is zero with silent relay nodes in Figure-8(b). The reason being overlap of relay transmission regions increases the number of nodes to be silent to double compared to non overlap region. When the throughput requirement is more, the time it takes to send more amount of data increases as the number of nodes in silent are more. If the relay nodes in the hoping region belong to overlapping region, these nodes remain silent instead of forwarding data to next hop. Thus in this section the effect of silent relay nodes on the end-to-end delay is extensively analysed.

\section{Conclusion}

End-to-end delay is one of the important quality of service metric considered in wireless adhoc network. Effect of transmission radius(r), channel access probability (p), node density $(\Lambda)$, and throughput $(\lambda)$ are studied in the literature. Effect of silent relay nodes delay in dense overlapping transmission regions is analysed in this paper. The simulation results show that the overlap of relay node transmission regions in dense network creates more end-to-end delays. The number of nodes to remain silent is increased to decrease the interference among nodes. End-to-end delay is analysed with respect to transmission $\operatorname{radius}(\mathrm{r})$, channel access probability(p) by considering node density $(\Lambda)$, throughput $(\lambda)$ and percentage of silent relay $\operatorname{nodes}(\alpha)$. Results show that end-to-end delay is more with silent relay nodes compared to without silent relay nodes. The node density and throughput parameters are varied one at a time to investigate the effect on end-to-end delay. The simulation results show that silent relay nodes which have common transmission region is affecting considerably the total end-to-end delay by reducing the optimal minimal region of end-to-end delay. The effect of node density $(\Lambda)$ is dominant with respect to throughput changes considering silent relay nodes as shown in the simulation results. The parameters considered for the analysis are interdependent. Exact expression for end-to-end delay by considering interdependencies among the parameters are future scope of research

\section{REFERENCES}

[1] Gomez J.and Campbell A.T., Naghshineh M., and C.Bisdikian, "Suporting dynamic power controlled routing in wireless adhoc networks", ACM/Kluwer Journal on Wireless Networks (WINET), to appear 2003.

[2] Abbas El Gamal, James Mammen, Balaji Prabhakar, and Devavrat Shah "Throughput-delay trade-off in wireless networks", Proc. INFOCOM, 2004.

[3] MGrossglauser and D Tse "Mobility increases the capacity of ad hoc networks", Proc.INFOCOM, 2001.

[4] Piyush Gupta and P.R.Kumar "The capacity of wireless networks",IEEE Transactions on Information Theory, March 2000.

[5] W. Heinzelman, A. Chandrakasan, and H. Balakrishnan "Energy-efficient communication protocol for wireless microsensor networks", In Proc. HICSS January 2000.

[6] Vikas Kawadia and P.R.Kumar "Power control and clustering in ad-hoc networks", Proc. INFOCOM, April 2003.

[7] K.Kar, M.Kodialam, T.V.Lakshmanan, and L.Tassiulas "Routing for network capacity maximization in energy-constrained adhoc networks", Proc. INFOCOM, April 2003.

[8] Srihari Narasimhan and Srisankar Kunniyur "Delay differentiation in sensor networks using power control", In Proc. CISS, March 2004.

[9] R. Rozovsky and P. R. Kumar SEEDEX: "A MACprotocol for ad hoc networks", In Proceedings of The ACM Symposium on Mobile Ad Hoc Networking and Computing, MobiHoc October 2001.

[10] G Sharma and R R Mazumdar "On achievable delay/capacity trade-offs in mobile ad hoc networks", Workshop on Modeling and Optimization in Mobile, Ad Hoc and Wireless Networks, March 2004.

[11] Suresh Singh and C.S.Raghavendra "Power efficient mac protocol for multihop radio networks", Proc IEEE International Symposium on Personal, Indoor and Mobile Radio Communications, 1998.

[12] Wei Ye, John Heidemann, and Deborah Estrin "An energy-efficient mac protocol for wireless sensor networks", Proc. of the 21st International Annual Joint Conference of the IEEE Computer and Communications Societies, June 2002

[13] Srihari Narasimhan and Srisankar Kunniyur Effect of network parameters on delay in wireless adhoc networks, August 29, 2004 